\title{
Priming and effector dependence on insulin B:9-23 peptide in NOD islet autoimmunity
}

\author{
Maki Nakayama, ${ }^{1}$ Joshua N. Beilke, ${ }^{1}$ Jean M. Jasinski, ${ }^{1}$ Masakazu Kobayashi, ${ }^{1}$ \\ Dongmei Miao, ${ }^{1}$ Marcella Li, ${ }^{1}$ Marilyne G. Coulombe, ${ }^{1}$ Edwin Liu, \\ John F. Elliott, ${ }^{2}$ Ronald G. Gill, ${ }^{1}$ and George S. Eisenbarth ${ }^{1}$
}

\author{
${ }^{1}$ Barbara Davis Center for Childhood Diabetes, University of Colorado Health Sciences Center (UCHSC), Aurora, Colorado, USA. \\ 2Department of Medical Microbiology and Immunology, Faculty of Medicine and Dentistry, University of Alberta, Edmonton, Alberta, Canada.
}

\begin{abstract}
NOD mice with knockout of both native insulin genes and a mutated proinsulin transgene, alanine at position B16 in preproinsulin (B16:A-dKO mice), do not develop diabetes. Transplantation of NOD islets, but not bone marrow, expressing native insulin sequences (tyrosine at position B16) into B16:A-dKO mice rapidly restored development of insulin autoantibodies (IAAs) and insulitis, despite the recipients' pancreatic islets lacking native insulin sequences. Splenocytes from B16:A-dKO mice that received native insulin-positive islets induced diabetes when transferred into wild-type NOD/SCID or B16:A-dKO NOD/SCID mice. Splenocytes from mice immunized with native insulin B chain amino acids 9-23 (insulin B:9-23) peptide in CFA induced rapid diabetes upon transfer only in recipients expressing the native insulin B:9-23 sequence in their pancreata. Additionally, $\mathrm{CD}^{+} \mathrm{T}$ cells from $\mathrm{B} 16: \mathrm{A}-\mathrm{dKO}$ mice immunized with native insulin $\mathrm{B}: 9-23$ peptide promoted IAAs in NOD/SCID mice. These results indicate that the provision of native insulin B:9-23 sequences is sufficient to prime anti-insulin autoimmunity and that subsequent transfer of diabetes following peptide immunization requires native insulin B:9-23 expression in islets. Our findings demonstrate dependence on B16 alanine versus tyrosine of insulin B:9-23 for both the initial priming and the effector phase of NOD anti-islet autoimmunity.
\end{abstract}

\section{Introduction}

In patients who develop organ-specific autoimmunity, an important question is why only certain organs are targeted (1-3). We believe that there may be single or multiple primary autoantigens that are specific to a target organ and trigger the autoimmune response, although there are examples of autoimmunity directed against autoantigens expressed in multiple tissues for some organspecific autoimmune diseases. In type $1 \mathrm{~A}$ diabetes, which is a pancreatic $\beta$ cell-specific autoimmune disease, insulin and the isletspecific glucose-6-phosphatase catalytic subunit-related protein (IGRP) have been identified as $\beta$ cell-specific autoantigens (4-6).

For type 1A (immune-mediated) diabetes, insulin has been proposed as a key autoantigen $(7,8)$. Insulin has been shown to be a target of both $\mathrm{T}$ and $\mathrm{B}$ lymphocytes with the demonstration of both insulin autoantibodies (IAAs) and insulin-reactive T cells in patients with type $1 \mathrm{~A}$ diabetes, in prediabetic subjects, and in animal models such as the NOD mouse (9-13). IAAs are frequently detected in sera of patients and NOD mice before and after diabetes onset $(14,15)$. Kent et al. recently reported that $\mathrm{CD}^{+} \mathrm{T}$ cell clones isolated from pancreatic lymph nodes of patients with type 1 diabetes react with an insulin A chain peptide (amino acids 1-15) restricted by DR4 (16). In NOD mice, both $\mathrm{CD}^{+}$and $\mathrm{CD}^{+} \mathrm{T}$ cells derived from pancreatic lymph nodes and pancreatic islets show insulin reactivity $(17,18)$, and insulin-reactive $\mathrm{T}$ cell clones established from pancreatic islets show cytotoxicity to pancreatic $\beta$ cells $(19,20)$. The observations that IAA levels are highest in the youngest children developing dia-

Nonstandard abbreviations used: B16:A, alanine at position 16 of insulin B chain; B16:A-dKO, double Ins1 and Ins2 knockout NOD mice with a mutated B16:A preproinsulin transgene; B16:Y, tyrosine at position 16 of insulin B chain; B16:Y-dKO, double Ins1 and Ins2 knockout NOD mice with a mutated B16:Y preproinsulin transgene; IAA, insulin autoantibody; insulin B:9-23, insulin B chain amino acids 9-23.

Conflict of interest: The authors have declared that no conflict of interest exists.

Citation for this article: J. Clin. Invest. 117:1835-1843 (2007). doi:10.1172/JCI31368. betes and usually precede the development of other autoantibodies $(21,22)$ and that insulin-reactive $\mathrm{T}$ cells are preferentially detected in younger NOD mice (23) have led to the hypothesis that insulin may be a crucial autoantigen in initiating islet autoimmunity. Supporting this hypothesis are the separate findings of Jaeckel et al. and French et al. that targeting $T$ cells reacting with insulin results in dramatic prevention of type 1 diabetes in $\operatorname{NOD}$ mice $(24,25)$.

Among the insulin epitopes recognized by NOD islet-infiltrating $T$ cells, insulin B chain amino acids 9-23 (insulin B:9-23) is reported to be a key peptide (26). Anti-insulin B:9-23 CD4+ TCR transgenes can induce (BDC12-4.1) (27) or prevent diabetes (2H6) (28). We recently reported that dual Ins1 and Ins2 knockout NOD mice that express a mutated insulin transgene - the wild-type tyrosine at amino acid 16 of the insulin B chain replaced with alanine - are protected from anti-islet autoimmunity and prevent diabetes (29). These mice lack both endogenous insulin genes and were rescued from an absolute insulin deficiency by a transgene expressing the alanine-to-tyrosine mutation in Ins 2 under control of the Rat insulin promoter. Mice carrying the transgene express an altered form of Ins2 (alanine at position 16 of insulin B chain; B16:A). These results suggest that insulin, and specifically the insulin B:9-23 sequence, may be essential for the initiation of the spontaneous diabetogenic autoimmune process of NOD mice.

Given the prevention of diabetes in NOD mice lacking native insulin B:9-23 sequences, one obvious question arises: In which tissues would expression of the native insulin sequence restore anti-insulin autoimmunity? Besides the target pancreatic $\beta$ cells, preproinsulin is reported to be expressed in thymic epithelial, thymic dendritic, and a subset of peripheral dendritic cells, potential sites at which preproinsulin expression may modulate insulin autoimmunity (30-32). With the creation of multiple NOD strains lacking native insulin genes, including strains with the NOD/SCID mutation, it is now possible to transplant islets 

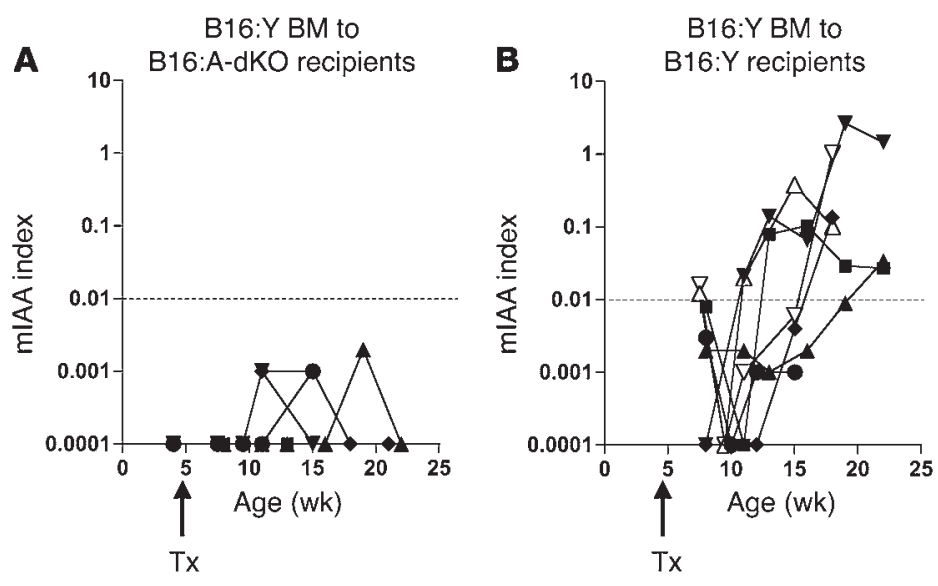

Figure 1

Bone marrow transplant with native $B 16: Y$ insulin genes is not sufficient to restore insulin autoimmunity. Four-week-old irradiated B16:A-dKO (A) or wildtype B16:Y mice $(B)$ were transplanted $(T x)$ with bone marrow derived from 4-week-old wild-type NOD mice. Native insulin-positive B16:Y bone marrow cells did not induce IAAs in B16:A-dKO mice but did induce IAAs in B16:Y mice. Each line represents an individual recipient. The $y$ axis represents the micro-IAA assay (mIAA) index in log scale.

or bone marrow with native (tyrosine at position 16 of insulin $\mathrm{B}$ chain; B16:Y) or altered B16:A insulin sequences to assess their influence on IAA production, insulitis, and diabetes. In this study we found that transplanted islet cells, but not bone marrow cells, expressing the native B16:Y insulin sequence restored anti-insulin autoimmunity in mice that lacked native insulin genes. In addition, immunization with the native $\mathrm{B} 16: \mathrm{Y}$ insulin $\mathrm{B}: 9-23$ peptide, but not the mutated B16:A insulin B:9-23 peptide, rendered $\mathrm{CD} 4^{+}$ $\mathrm{T}$ cells able to rapidly transfer anti-insulin autoimmunity and diabetes to NOD/SCID mice when the recipient mouse also had the native $\mathrm{B} 16: \mathrm{Y}$ insulin $\mathrm{B}: 9-23$ sequence in its islets.

\section{Results}

Transplantation of bone marrow with native insulin genes does not restore insulin autoimmunity. Preproinsulin is reported to be expressed in thymic epithelial, thymic dendritic, and a subset of peripheral dendritic cells (30-32). In order to determine whether native insulin gene expression in hematopoietic cells is sufficient to induce anti-islet autoimmunity, $1 \times 10^{7}$ bone marrow cells derived from 4-week-old wild-type B16:Y NOD mice were transplanted into irradiated 4-week-old double Ins1 and Ins2 knockout mice with a mutated B16:A preproinsulin transgene (B16:A-dKO mice). None of the 5 B16:A-dKO recipients developed IAAs (Figure 1A). In contrast, 6 of 7 B16:Y NOD recipients of wild-type B16:Y bone marrow expressed anti-IAAs (Figure 1B). Thus, bone marrow cells carrying the native B16:Y insulin sequence do not restore anti-insulin autoimmunity in B16:A-dKO mice.

Transplantation of native B16:Y insulin-bearing islets induces development of IAAs. Using islet transplantation, we sought to determine whether native insulin expression in transplanted islets is sufficient to induce anti-islet autoimmunity in B16:A-dKO mice. Two hundred islets from native B16:Y insulin-bearing NOD/SCID mice were transplanted under the kidney capsule of 4-week-old B16:AdKO mice. High IAA levels developed within 3 weeks of transplantation in 11 of $13 \mathrm{~B} 16: \mathrm{Y}$ islet recipients (Figure 2A). In contrast, 1 of 8 mice transplanted with B16:A-dKO islets developed IAAs after transplant, and only at a low titer $(P<0.0001$; Figure 2B). Therefore, islet expression of insulin with the native insulin B:9-23 sequence, even with ectopic expression under the kidney capsule, is sufficient to rapidly induce IAAs in NOD mice lacking native insulin B:9-23 sequences.

Induction of insulitis by B16:Y islet transplant. The B16:Y islet grafts (Figure 3, A and C), but not the B16:A-dKO islet grafts (Figure 3, B and D), were completely destroyed by lymphocytic infiltration. This suggests that wild-type insulin, with the native B16:Y insulin B:9-23 sequence, in transplanted islet grafts is recognized by lymphocytes of B16:A-dKO mice and that these lymphocytes are able to kill the native insulin-positive islet cells. In addition to analyzing the histology of the transplanted islet grafts, we also analyzed insulitis of the endogenous pancreatic islets of the $\mathrm{B} 16: \mathrm{A}-\mathrm{dKO}$ recipient mice. All 5 recipients of B16:Y islets that were sacrificed fewer than 20 weeks after transplant showed insulitis (Figure 3E) but not recipients of B16:A islets (Figure 3F). Pancreatic insulitis, however, was transient: 5 of 10 mice receiving the same $\mathrm{B} 16$ : $\mathrm{Y}$ islet transplants that were sacrificed more than 35 weeks after islet transplant had insulitis. Insulitis at less than 20 weeks after transplant was significantly more severe than at more than 30 weeks after transplant $\left(P=0.02, \chi^{2}\right.$ test; Figure $\left.3 G\right)$. Pancreatic insulitis of $\mathrm{B} 16: \mathrm{A}-\mathrm{dKO}$ mice receiving $\mathrm{B} 16: \mathrm{Y}$ islets was more severe than that of mice receiving B:16A-dKO islet grafts $\left(P<0.03, \chi^{2}\right.$ test; Figure $3 \mathrm{G})$. Importantly, insulitis of mice receiving B16:A-dKO islets did not differ from unmanipulated B16:A-dKO mice, which did not receive islet transplants. These results indicate that transient insulitis of the B16:A pancreas induced by islet transplants is specific to the native $\mathrm{B} 16: \mathrm{Y}$ insulin sequence of donor islets.

While transplantation of $\mathrm{B} 16: \mathrm{Y}$ islets into $\mathrm{B} 16: \mathrm{A}-\mathrm{dKO}$ mice induced not only graft destruction but also lymphocytic infiltration of the recipients' pancreatic islets, diabetes was not induced. None of the B16:A-dKO mice that received B16:Y islet transplants became diabetic (0 of 11, followed up to 35 weeks). Even though insulitis was induced in the host pancreas, it was
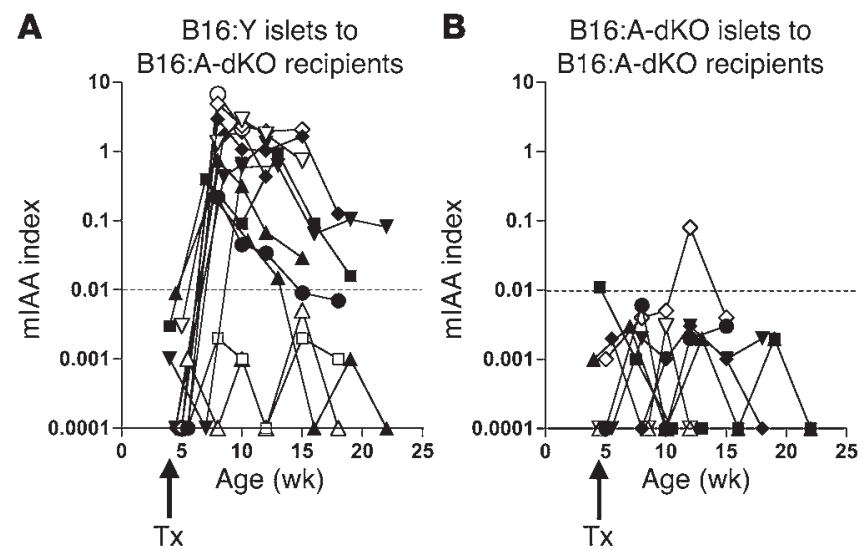

Figure 2

Development of IAAs after B16:Y NOD/SCID islet transplant. (A) B16: A-dKO mice, when transplanted with native B16:Y insulin NOD/SCID islets, developed IAAs. (B) Mice receiving B16:A-dKO islets did not express insulin antibodies after transplant, with the exception of 1 mouse. Each line represents an individual mouse. The $y$ axis represents the micro-IAA assay index in log scale. 
B16:Y islets to B16:A-dKO recipients

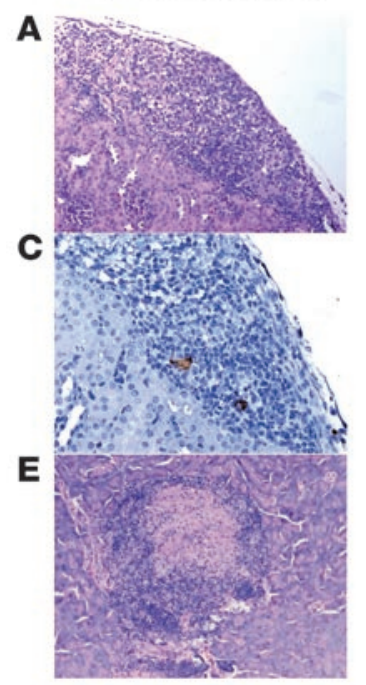

B16:A-dKO islets to B16:A-dKO recipients

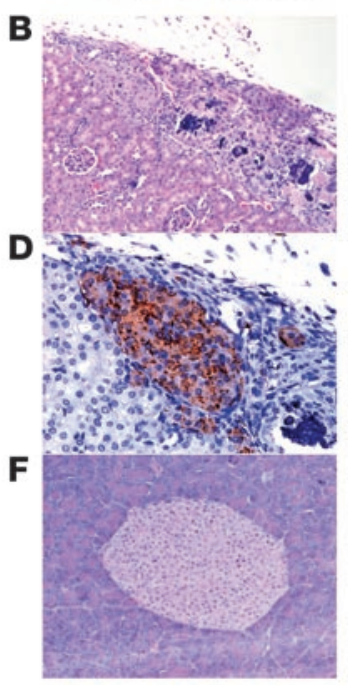

G

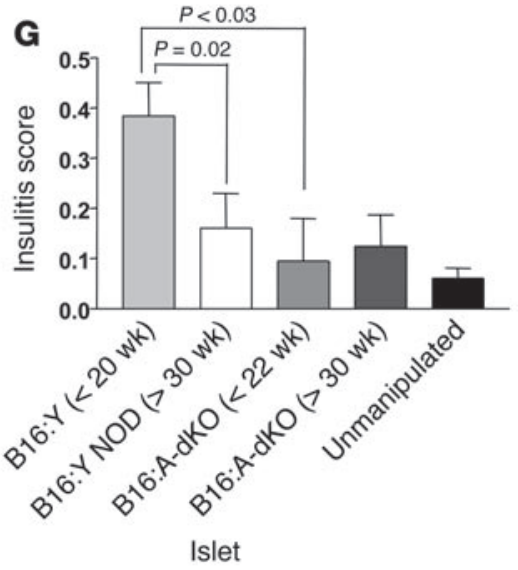

Figure 3

Native B16:Y islet transplants induce graft insulitis and severe transient insulitis in endogenous pancreatic islets. (A-D) B16:Y NOD/SCID (A and C) and B16:A-dKO (B and $\mathbf{D})$ islets were transplanted under the kidney capsule of B16:A-dKO mice. (A and B) H\&E stain. (C and D) Insulin stain. Two weeks after transplant, the B16:Y NOD/SCID islet graft showed very little insulin staining and severe lymphocytic infiltration, whereas the B16:A-dKO islet graft was intact. (E and F) Endogenous pancreatic islets of B16: $Y$ islet recipients $(E)$, but not B16:A-dKO islet recipients $(\mathbf{F})$, showed marked lymphocytic infiltration 18 weeks after islet transplant. Original magnification, $\times 100$ (A, B, E, and F); $\times 200$ (C and D). (G) More than 10 pancreatic islets from each B16:A-dKO mouse receiving B16:Y NOD/SCID or B16:A-dKO islet transplant were evaluated for lymphocytic infiltration less than 20 weeks or more than 30 weeks after transplant $(n=5-10)$. Pancreatic islets from an age-matched unmanipulated B16:A-dKO mouse were also evaluated. The $y$ axis represents the mean \pm SD of the insulitis score.

not sufficient to cause diabetes in mice whose pancreatic islets lacked the native insulin B:9-23 sequence.

Splenocytes from B16:A-dKO recipients of native B16:Y insulin-bearing islet transplants are diabetogenic. In order to evaluate whether lymphocytes from B16:A-dKO mice with B16:Y NOD/SCID islet transplant can cause diabetes if the host pancreas has the native B16:Y insulin sequence, we transferred splenocytes from B16:A-dKO mice that had received islet transplants into wild-type B16:Y NOD/SCID or B16:A-dKONOD/SCID mice(Figure 4A).Splenocytes from B16:A-dKO mice transplanted with $\mathrm{B} 16: \mathrm{YNOD} / \mathrm{SCID}$ islets rapidly induced diabetes in B16:Y NOD/SCID mice (5 of 6; Figure 5A), which indicates that immunization with B16:Y islets generates splenocytes able to recognize and destroy B16:Y pancreatic islets (i.e., induces autoimmunity) and that B16:A-dKO recipients of B16:Y islets contain functional native insulin-autoreactive lymphocytes in the absence of disease. In contrast, when splenocytes were transferred from B16:A-dKO mice that received B16:A-dKO islets, diabetes occurred in 2 of 8 B16: $Y$ NOD/SCID recipients, at a later time after splenocyte transfer $(P<0.01$; Figure $5 \mathrm{~A})$. This is potentially consistent with the natural late (i.e., independent of islet transplant) priming that occurred after transfer of splenocytes into a B16:Y NOD/SCID host from unmanipulated B16:A-dKO mice (Figure 5A). In the opposing model, in which the splenocyte recipients were B16:A-dKO NOD/SCID mice, diabetes occurred in 2 of 5 mice receiving splenocytes from B16:Y islet recipients (Figure 5B). In contrast, splenocytes from B16:A-dKO mice transplanted with B16:A-dKO islets did not induce diabetes or insulitis upon transfer to B16:A-dKO NOD/SCID mice (Figure 5B).

These results indicate that the diabetogenic splenocytes are induced by B16:Y islet transplants, which can destroy $\beta$ cells in NOD/SCID mice with either $\mathrm{B} 16: \mathrm{Y}$ or B16:A pancreatic islets. Yet, B16:A islet transplants are unable to generate diabetogenic lymphocytes, for diabetes did not develop whether the recipient NOD/SCID mouse had insulin with B16:A or B16:Y. This is consistent with the importance of the native insulin $\mathrm{B}: 9-23$ sequence in activating diabetogenic anti-islet autoimmunity.

Immunization with insulin B:9-23 peptidesinduces IAAsbutnotinsulitis. To determine whether the provision of just the insulin B:9-23 peptide rather than islets with $B 16$ : $A$ versus $B 16: Y$ insulin is sufficient to restore anti-islet autoimmunity and diabetogenicity, we immunized 4-week-old B16:A-dKO mice with $100 \mu \mathrm{g}$ of B16:Y insulin B:9-23 peptide or B16:A insulin B:9-23 peptide in CFA (Figure 4B). More than 95\% (29 of 30) of B16:A-dKO mice immunized with B16:Y insulin B:9-23 peptide developed antiIAAs (data not shown). These antibodies were not absorbed by control tetanus toxin peptide or B:9-23 peptides, but were absorbed by insulin (Figure 6A). The absorbing insulin contained the $\mathrm{B} 16: \mathrm{Y}$ insulin $\mathrm{B}: 9-23$ sequence; therefore, insulin is an autoantigen corresponding to this peptide. All B16:A-dKO mice (15 of 15) immunized with the B16:A insulin B:9-23 peptide similarly produced IAAs that were not absorbed by the peptides, but were absorbed by insulin (Figure 6B). As expected, B16:Y NOD mice immunized with B16:Y insulin B:9-23 peptide also produced IAAs (Figure 6C).

Despite the induction of IAAs, immunization with both insulin B:9-23 peptides did not induce lymphocytic infiltration of pancreatic islets analyzed 10-15 weeks after immunization (Figure 6E). This suggests that provision of the insulin B:9-23 peptide itself is insufficient to induce inflammation of endogenous pancreatic islets lacking the B16:Y insulin B:9-23 sequence. Correlating with the lack of insulitis, none of the mice progressed to diabetes.

Immunization with B16:Y insulin B:9-23 peptide-induced diabetogenic splenocytes induces diabetes in NOD/SCID recipients. As outlined in Figure $4 \mathrm{~B}$, immunization with insulin $\mathrm{B}: 9-23$ peptides did not induce insulitis in B16:A-dKO mice. Nevertheless, when we transferred splenocytes from B16:A-dKO mice immunized with B16:Y peptide, recipient B16:Y NOD/SCID mice, but not B16:A-dKO NOD/SCID mice, developed IAAs and diabetes. As shown in Figure 6D and 7A, splenocytes from B16:A-dKO mice immunized with B16:Y insulin B:9-23 peptide induced high levels of IAAs within 10-20 days of transfer to B16:Y NOD/SCID mice. Splenocytes from B16:A-dKO mice immunized with B16:A insulin B:9-23 peptide did not rap- 
A
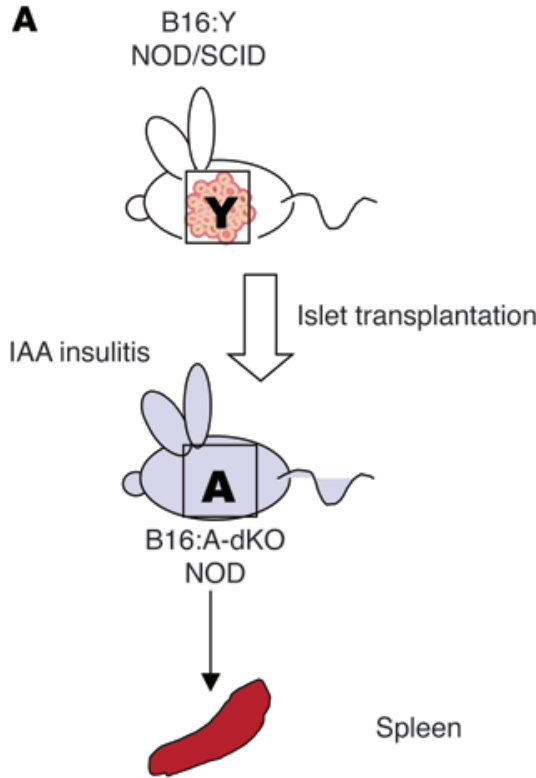

B16:A-dKO NOD
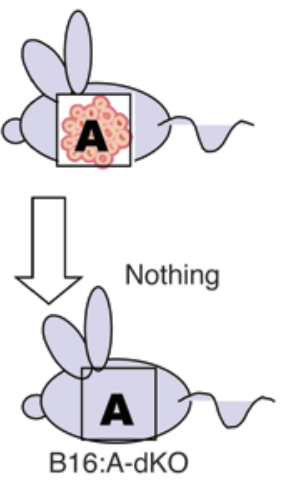

NOD

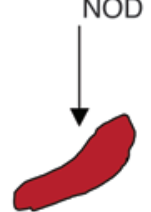

B

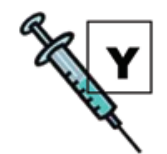

B16:Y B:9-23

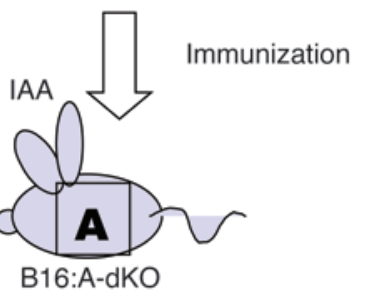

NOD

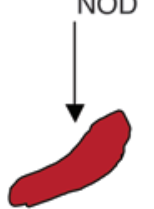

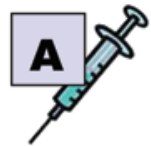

B16:A B:9-23

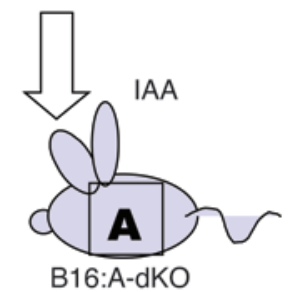

NOD

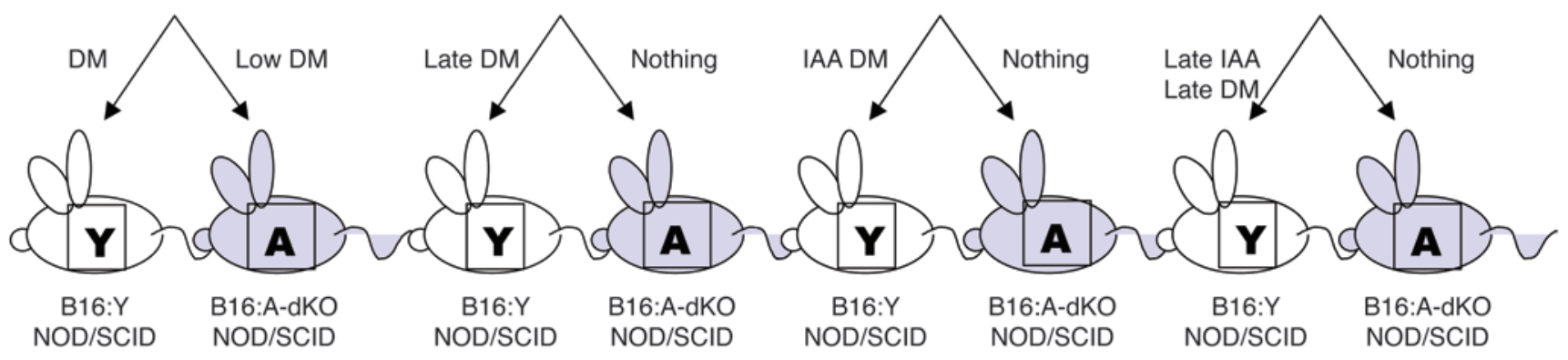

Figure 4

Summary of experiments with transfer of splenocytes into NOD/SCID recipients for mice immunized with islets (A) or insulin B:9-23 peptides (B). DM, diabetes mellitus.

idly induce IAAs in B16:Y NOD/SCID (Figure 7C) or B16:A-dKO NOD/SCID recipients (Figure 7D). Even splenocytes from mice immunized with B16:Y insulin B:9-23 peptide did not rapidly induce IAAs in B16:A-dKO NOD/SCID mice (Figure 7B).

As shown in Figure 8, only the combination of immunization with B16: $\mathrm{Y}$ insulin B:9-23 peptide in B16:A-dKO mice followed by splenocyte transfer to B16:Y NOD/SCID mice caused diabetes in the recipient. In contrast, these same splenocytes did not induce diabetes in the B16:A-dKO NOD/SCID recipients, which suggests that pancreatic islet expression of native B16:Y insulin is necessary for diabetes following immunization with just the peptide in contrast to immunizing with (i.e., transplanting) whole islets. Splenocytes from mice immunized with B16:A insulin B:9-23 peptide did not rapidly transfer diabetes in B16:Y NOD/SCID mice (2 of 8 mice developed diabetes at 65 and 110 days after transfer; Figure 8; as seen in B16:Y recipients of splenocytes from nonimmunized mice; see Figure $5 \mathrm{~A}$ ). Taken together, our results suggest the 2 requirements for efficient insulin B:9-23 peptide induction of diabetes are priming by the B16: Y insulin B:9-23 sequence and splenocytes encountering native B16: $Y$ insulin in the pancreatic islets of the recipient.

B16:Y insulin B:9-23-primed $C D 4^{+} T$ cells are sufficient to transfer IAA production. Immunization of $\mathrm{B} 16: \mathrm{A}-\mathrm{dKO}$ splenocyte donors with native $B 16: Y$ peptide is essential for the rapid induction of IAAs after splenocyte transfer to B16:Y NOD/SCID mice. Because the spleen contains $\mathrm{CD} 4^{+} \mathrm{T}$ cells and non-CD4 $4^{+}$splenic cells (e.g., macrophages, B lymphocytes, and dendritic cells) that may take up B16:Y peptide, we set out to determine which of these subsets of cells were responsible for inducing IAA production. We transferred $\mathrm{CD}^{+} \mathrm{T}$ cells isolated from the spleens of B16:A-dKO mice immunized with B16:Y insulin B:9-23 peptide to NOD/SCID mice. Because NOD/SCID mice lack B lymphocytes, we also simultaneously transferred CD4-depleted splenocytes derived from nonimmunized B16:A-dKO mice. As shown in Figure 9A, splenic CD4+ $\mathrm{T}$ cells from the insulin B:9-23 peptide-immunized mice with non$\mathrm{CD}^{+}$cells from nonimmunized mice upon transfer were sufficient to induce IAAs. In contrast, transfer of non-CD4 ${ }^{+}$splenocytes from immunized mice together with $\mathrm{CD}^{+}$splenocytes from nonimmunized mice failed to induce IAAs (Figure 9B), even though the immunized donor mice had IAAs. These results indicate that the induction of IAAs after splenocyte transfer is dependent on $\mathrm{CD}^{+} \mathrm{T}$ cells from the B16:Y insulin B:9-23 peptide-immunized mouse and non-CD4 ${ }^{+}$splenocytes do not induce anti-IAAs upon transfer.

Provision of the B16:Y insulin B:9-23 sequence by transgenesis induces IAAs and insulitis. In order to explore whether induction of IAAs following provision of $\mathrm{B} 16: \mathrm{Y}$ insulin $\mathrm{B}: 9-23$ sequences to a $\mathrm{B} 16: \mathrm{A}-\mathrm{dKO}$ mouse is dependent upon postnatal B:9-23 exposure, we evaluated an additional model for induction of IAAs. We created double Ins1 and Ins2 knockout NOD mice with a mutated B16:Y preproinsulin transgene 
A

B16:Y NOD/SCID recipient

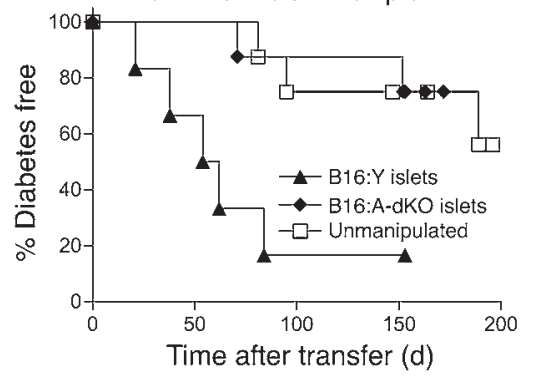

B B16:A-dKO NOD/SCID recipient

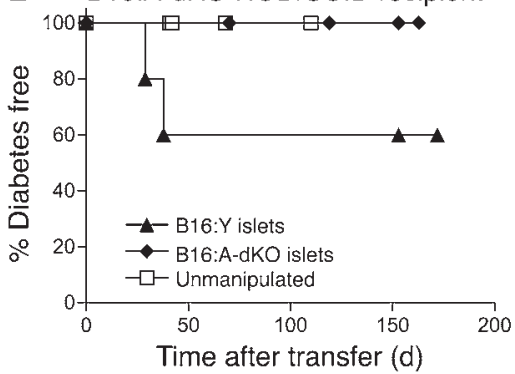

Figure 5

Rapid induction of diabetes with splenocytes from mice transplanted with B16:Y islets transferred into NOD/SCID mice with B16:Y. Splenocytes from B16:A-dKO mice that received $B 16: Y N O D / S C I D$ islets, $B 16: A-d K O$ islets, or no transplant (unmanipulated) were transferred into wild-type B16:Y NOD/SCID mice (A) or B16:A-dKO NOD/SCID mice (B). $P<0.01, \mathrm{~B} 16: \mathrm{Y}$ islets versus B16:A-dKO islets in B16:Y NOD/SCID recipients.

(B16:Y-dKO mice) driven off the same promoter as our mutated B16: A preproinsulin transgene. Introducing this transgene with the $B 16: Y$ insulin B:9-23 sequence into B16:Y-dKO mice resulted in expression of IAAs (Figure 10A). More than half of B16:Y-dKO mice (5 of 10) developed high levels of IAAs, whereas 2 of 31 B16:A-dKO mice developed IAAs at low levels $(P<0.01)$. In addition, B16:Y-dKO mice devel- oped insulitis as severe as that of age-matched wildtype NOD mice, whereas age-matched B16:A-dKO mice did not $(P<0.01$; Figure 10, B-D). We evaluated mice for islet infiltration between 10 and 22 weeks of age. Most B16:A-dKO mice did not have any lymphocytic infiltration, and 2 of 9 mice had peri-islet infiltration, which was observed in less than $20 \%$ of the islets in individual mice. In contrast, all of the $4 \mathrm{~B} 16: \mathrm{Y}-\mathrm{dKO}$ mice developed severe insulitis, and $50 \%-70 \%$ of the islets demonstrated intraislet infiltration. These results indicate that even genetically induced expression of $\mathrm{B} 16: \mathrm{Y}$ proinsulin restores anti-islet autoimmunity as well as postnatal provision via islet transplant.

\section{Discussion}

We have evaluated 3 phenotypes (anti-IAA, insulitis, and development of diabetes) in multiple NOD-derived animal models by using varying combinations of islet transplants with different insulin B:9-23 sequences and corresponding insulin B:9-23 sequences in the recipient pancreatic islets (Tables 1 and 2). Our results highlight a dramatic dependence on the presence or absence of the native B16:Y insulin sequence, consistent with the hypothesis that the insulin B:9-23 peptide is a key determinant of NOD autoimmune diabetes and that
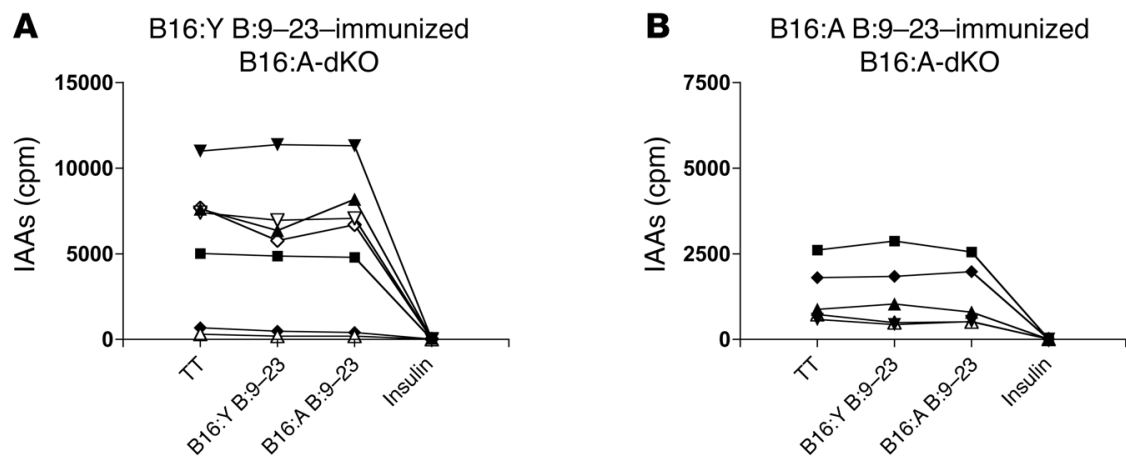

C

B16:Y B:9-23-immunized B16:Y

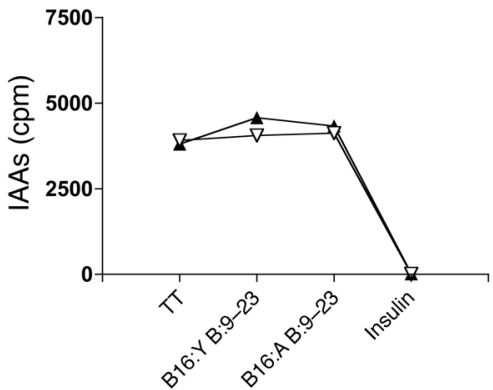

D B16:Y B:9-23-immunized B16:A-dKO splenocytes to B16:Y NOD/SCID recipients

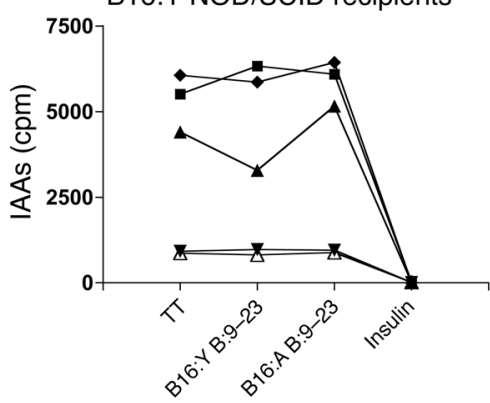

E

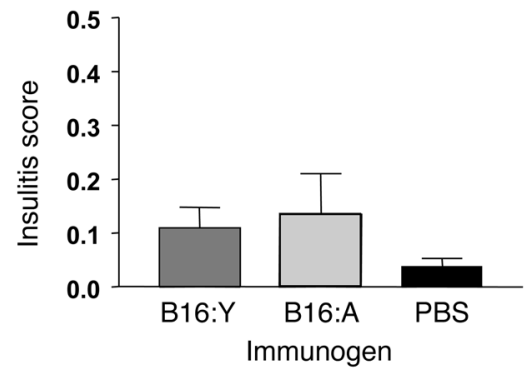

Figure 6

Development of IAAs but not insulitis by immunization with insulin B:9-23 peptide. (A-D) Serum from B16:A-dKO mice immunized with native $B 16: Y$ insulin $B: 9-23$ peptide (A), B16:A-dKO mice immunized with altered B16:A insulin B:9-23 peptide (B), wild-type B16:Y NOD mice immunized with native $B 16: Y$ insulin B:9-23 peptide (C), and wild-type B16:Y NOD/SCID mice that received splenocytes from insulin B:9-23 peptide-immunized B16:A-dKO mice (D) was incubated with I'25-insulin in the presence of tetanus toxin peptide (TT), native B16:Y insulin B:9-23 peptide, B16:A insulin B:9-23 peptide, or human insulin. Each line represents an individual mouse. (E) More than 10 pancreatic islets from each B16:A-dKO mouse immunized with B16:Y insulin B:9-23 peptide $(n=10)$, B16:A insulin B:9-23 peptide $(n=4)$, or PBS $(n=10)$ in CFA were evaluated for lymphocytic infiltration. The $y$ axis represents the mean $\pm S D$ of the insulitis score. 
A

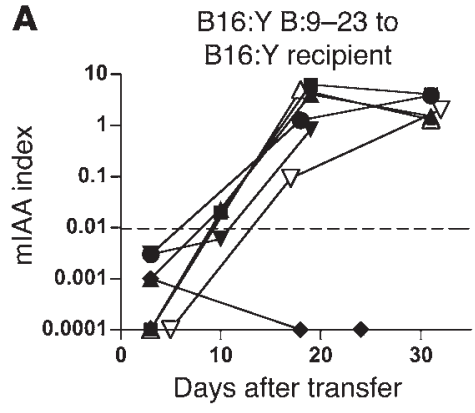

C

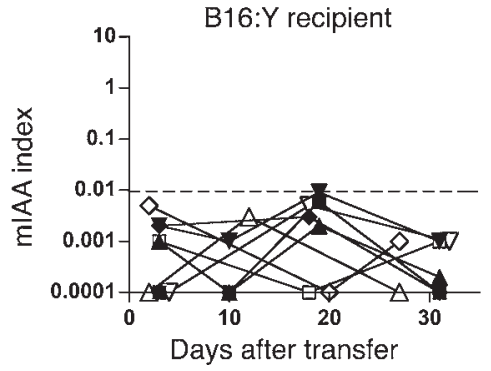

B
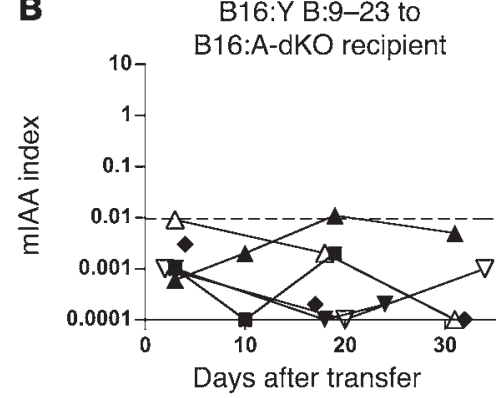

D

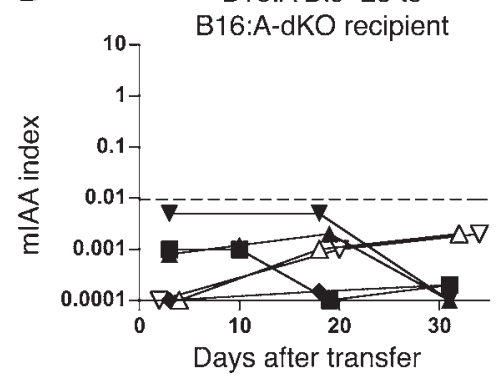

Figure 7

Only wild-type B16:Y NOD/SCID mice receiving splenocytes of B16:A-dKO mice immunized with native $B 16: Y$ insulin $B: 9-23$ peptide rapidly produce IAAs. Splenocytes from B16:A-dKO mice immunized with native insulin B:9-23 peptide (A and $\mathbf{B}$ ) or mutated B16:A insulin B:9-23 peptide ( $\mathbf{C}$ and $\mathbf{D}$ ) were transferred to wild-type $B 16$ : Y NOD/SCID (A and C) or B16:A-dKO NOD/SCID mice (B and D). IAAs were measured weekly after splenocyte transfer. Each line represents an individual mouse.

islet expression of the native insulin sequence, but not expression in bone marrow-derived cells, induces anti-islet autoimmunity. Even transgenic provision of the B16:Y insulin B:9-23 sequence restored IAAs and insulitis in B16:A-dKO mice. Thus, postnatal exposure to the native $\mathrm{B} 16: \mathrm{Y}$ proinsulin sequence in $\mathrm{B} 16: \mathrm{A}-\mathrm{dKO}$ mice was not essential for inducing anti-islet autoimmunity, although it is possible that postnatal exposure may enhance the immune response. It is noteworthy that islets differing at a single amino acid of the insulin molecule (i.e., B16:Y versus B16:A) differed in their ability to induce diabetogenic T lymphocytes (Tables 1 and 2 ).

Transplantation of native insulin-positive bone marrow cells did not induce the development of IAAs in B16:A-dKO mice. This suggests that native insulin expression in hematopoietic cells, especially antigen-presenting cells such as dendritic cells, may not be sufficient to initiate anti-insulin autoimmunity. However, there is a caveat that the level of insulin expression in such hematopoietic cells may not have been sufficient to break tolerance compared with insulin expression in islets.

It was easier to induce IAAs than insulitis in B16:A-dKO mice, and diabetes only occurred upon transfer of splenocytes from immunized B16:A-dKO hosts into NOD/SCID mice. Thus, simply immunizing B16:A-dKO mice with islets or peptide with the B16: Y insulin B:9-23 sequence was not sufficient to induce diabetes (Table 1), which was not surprising given the lack of the B16:Y insulin B:9-23 sequence in the pancreatic islets of the recipient mouse. Of note, only transplantation of islets bearing the B16:Y insulin B:9-23 sequence, but not immunization with the B16:Y insulin B:9-23 peptide, induced insulitis. Islets may be more efficient inducers of pathogenic $T$ cells than peptides for many reasons. Islet transplant provides the whole preproinsulin molecule
(33), other antigens such as IGRP $(5,34,35)$, and perhaps specific danger signals related to the surgical procedure not provided by CFA (36). As shown in Table 2, when transferring splenocytes from islet-transplanted mice to NOD/SCID recipients, induction of insulitis and rapid diabetes required donor islets to have the B16: $Y$ insulin B:9-23 sequence, and induction of diabetes with peptide immunization required the B16:Y insulin B:9-23 sequence in both the peptide and the NOD/SCID recipient. Thus, priming by B16:Y insulin B:9-23 was sufficient to initiate anti-islet autoimmunity for disease transfer to NOD/SCID, and diabetes induction was specific to the B16:Y insulin B:9-23 sequence in pancreatic islets. We believe it is likely that in addition to $\mathrm{CD}^{+} \mathrm{T}$ cells, $\mathrm{CD} 8^{+} \mathrm{T}$ cells participate in the generation of diabetes as shown through the previously described insulin B:15-23 CD8 epitope (37).

Our studies indicate that it is $\mathrm{CD} 4^{+} \mathrm{T}$ cells from the splenocytes of immunized donor mice that transfer production of IAAs to NOD/SCID recipients. CD $4^{+}$ $\mathrm{T}$ cells from a nonimmunized splenocyte donor were unable to induce IAAs in NOD/SCID recipients. AntiIAAs appeared within 20 days, but only when the recipient mouse islets had the $\mathrm{B} 16: \mathrm{Y}$ insulin $\mathrm{B}: 9-23$ sequence. The same was true for the rapid induction of diabetes with insulin B:9-23 peptide immunization and splenocyte transfer. The speed with which IAAs appeared suggests that B lymphocytes of even the nonimmunized B16:A donor mice exist, awaiting nontolerant $\mathrm{CD}^{+}{ }^{+} \mathrm{T}$ lymphocytes (i.e., B16:Y primed) to help rapidly produce IAAs. Alternatively, the transferred B lymphocytes may rapidly acquire insulin with native insulin B:9-23 sequences from the B16:Y NOD/SCID host (38). Of note, the IAAs were not simply antibodies to the insulin $\mathrm{B}: 9-23$ peptide and were not absorbed with the peptide, but were absorbed with intact insulin, similar to the IAAs of regular NOD mice or NOD and Balb/c mice directly immunized with B:9-23 peptide (39). The ability of $\mathrm{CD}^{+} \mathrm{T}$ cells from B16:Y insulin B:9-23-immunized splenocyte donors alone to induce IAAs and diabetes should provide an assay to further define these pathogenic helper T lymphocytes.

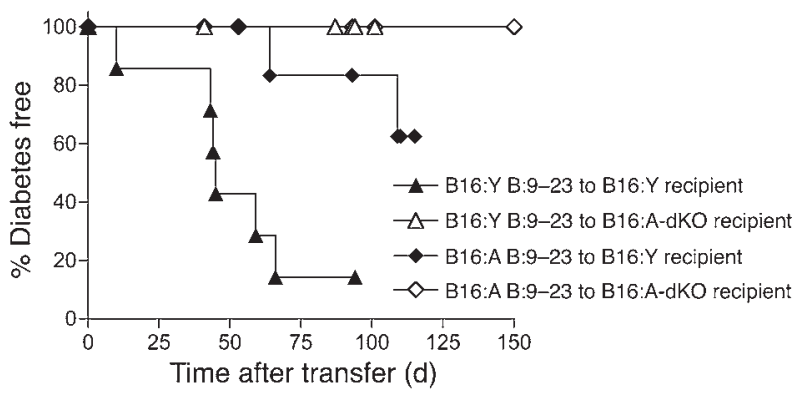

\section{Figure 8}

Development of diabetes after splenocyte transfer from insulin B:9-23 peptide-immunized B16:A-dKO mice. Splenocytes from mice immunized with native $B 16: Y$ insulin $B: 9-23$ peptide, but not with mutated $B 16: A$ insulin $B: 9-23$ peptide, rapidly transferred diabetes to wild-type $B 16: Y$ NOD/SCID mice. B16:A-dKO NOD/SCID mice did not develop diabetes following transfer of splenocytes from mice immunized with either native B16:Y insulin B:9-23 or mutated B16:A insulin B:9-23 peptides. 

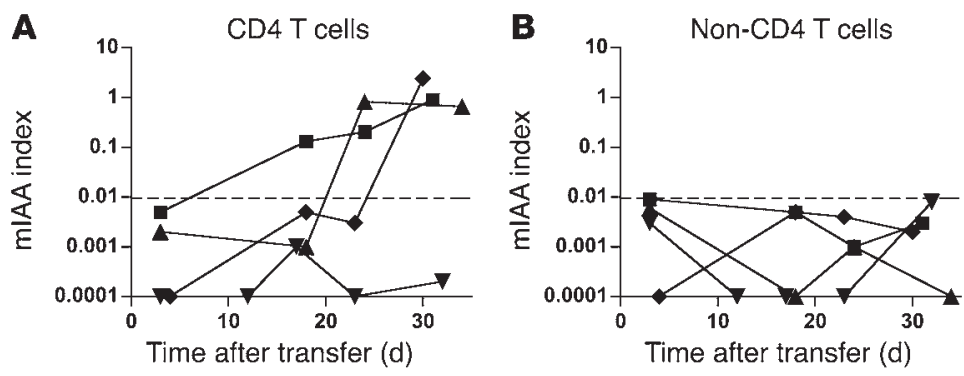

\begin{abstract}
Figure 9
Development of IAAs in wild-type B16:Y NOD/SCID mice after splenocyte transfer. (A) Splenic CD4 ${ }^{+} \mathrm{T}$ cells from B16: A-dKO mice immunized with native B16:Y insulin B:9-23 peptide were transferred to wild-type B16:Y NOD/SCID mice along with non-CD4 ${ }^{+}$splenocytes from unmanipulated double insulin-knockout mice. (B) Splenic CD4+ T cells from unmanipulated mice were transferred to wild-type B16:Y NOD/SCID mice along with a non-CD4+ splenocyte population from mice immunized with $B 16: Y$ insulin B:9-23 peptide. Each line represents an individual mouse.
\end{abstract}

A potentially unique feature of the insulin B:9-23 peptide is that TCR recognition of this epitope appears to be primarily determined by specific conserved $V_{\alpha}$ and $J_{\alpha}$ sequences without defined conservation of the $\mathrm{N}$ region of the $\alpha$ chain or TCR $\beta$ chain (40). Recognition with such a simplified TCR motif raises the possibility that there may be a high precursor frequency of thymic $\mathrm{T}$ cells reacting with insulin B:9-23. Insulin is a molecule that circulates at low concentrations but is expressed at extremely high concentrations within pancreatic $\beta$ cells (41). The general hypothesis we are pursuing is that insulin B:9-23 peptide is recognized by a low-stringency common TCR, which increases the ease of breaking $\mathrm{CD} 4^{+} \mathrm{T}$ cell tolerance to insulin, and that such loss of tolerance leads to production of IAAs and specific $\beta$ cell destruction.

Our present results indicate that loss of tolerance to the B16:Y insulin B:9-23 sequence at sites distinct from the pancreas and pancreatic lymph node (i.e., renal capsule or subcutaneous sites) can rapidly engender diabetogenic $\mathrm{T}$ lymphocytes and anti-IAAs. This raises the possibility that environmental mimotopes of such a peptide may contribute to diabetes by activating $\mathrm{CD}^{+} \mathrm{T}$ cell reactivity to a primary autoantigen. Once such tolerance to insulin or other islet molecules is lost, additional autoantigens are likely to be targeted. Whether there are additional epitopes of insulin or other molecules as central to the development of diabetes in the NOD mouse warrants further study. At present, it is unknown whether there exists a similar dominant epitope of insulin or another islet molecule for human type 1 diabetes. Our findings suggest that it may be fruitful to search for such a determinant, especially in individuals with a fixed HLA, such as DR3/DR4 heterozygous diabetics, which comprise $30 \%-50 \%$ of patients with type 1 diabetes.

\section{Methods}

Mice. B16:A-dKO mice were established as previously described (42). Briefly, Ins1 and Ins 2 knockout NOD mice were separately established by breeding the original insulin knockouts (kindly provided byJ.Jami, INSERM, Paris, France; ref. 43) onto NOD/Bdc mice using speed congenic techniques (44). Mutated B16:A NOD mice were produced by microinjection of mutated B16:A Ins2 cDNA constructs ligated to rat insulin 7 promoter (pRIP7) directly into NOD fertilized eggs (42). Ins1 knockouts, Ins2 knockouts, and B16:A NOD mice were combined and genotyped for the native insulin genes and the mutated insulin transgene (42). Native B16:Y Ins2 cDNA constructs ligated to pRIP7 were created by directly replacing nucleotides (GCC for alanine) in the vector carrying B16:A transgene with TAC for tyrosine using site-directed mutagenesis (Stratagene) and were microinjected into NOD fertilized eggs. B16:Y-dKO mice were established in the same manner as B16:A-dKO mice (42). NOD/SCID mice (NOD.CB17-Prkdcsid/J) were purchased from The Jackson Laboratory (stock no. 001303). B16:A-dKO mice were crossed with NOD/SCID mice, and (B16:A-dKO $\times$ NOD/SCID) F1 mice were intercrossed to obtain B16:A-dKO NOD/SCID mice. The NOD/SCID mutation was confirmed by PCR amplification of extracted genomic DNA (forward primer, GACTAGAAAGCTAGAGAGCT; reverse primer, AGTTATAACAGCTGGGTTGGC) followed by incubation with Alu I (Invitrogen) at $37^{\circ} \mathrm{C}$ for 2 hours. A final product of $239 \mathrm{bp}$ was considered wild type; the NOD/SCID mutation was $209 \mathrm{bp}$.

Only female mice were used for experiments. All mice were bred and housed in a pathogen-free animal colony at the UCHSC Center for Comparative Medicine. All experiments were approved by the University of

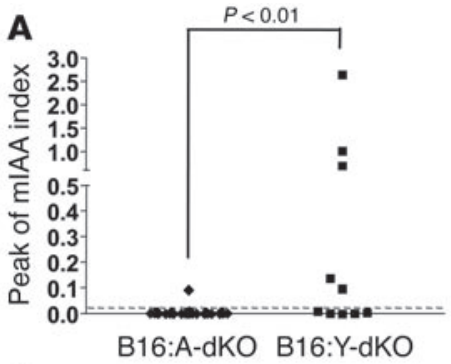

C

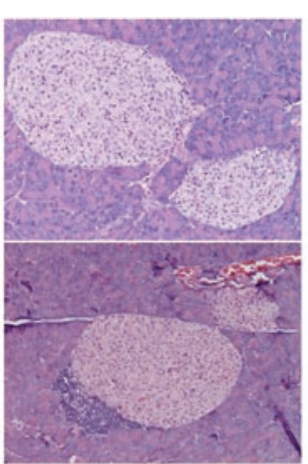

B

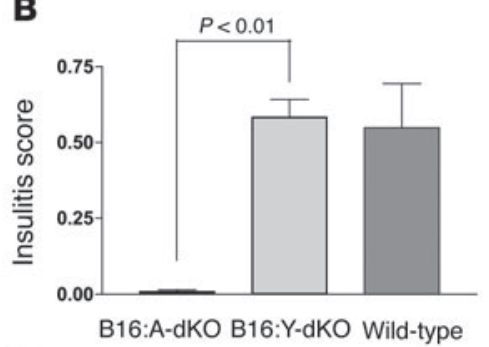

D

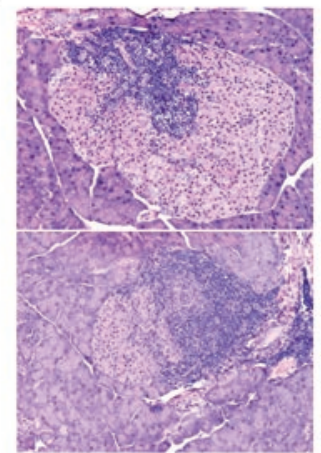

\section{Figure 10}

Provision of the native $B 16: Y$ insulin $B: 9-23$ sequence by transgenesis induces IAAs and insulitis. (A) B16:A-dKO and B16:Y-dKO mice were measured for the development of IAAs every 2-3 weeks between 4 and 30 weeks of age. Each symbol represents the peak level of mIAA index for individual mice. B16:Y-dKO mice developed IAAs $(P<0.01$ versus B16:A-dKO). (B) Insulitis scoring of B16:A-dKO mice, B16:Y-dKO mice, and wild-type NOD mice between 10 and 22 weeks of age. B16:Y-dKO mice developed insulitis significantly more severe than did B16:A-dKO mice $(P<0.01)$ and as severely as did wild-type NOD mice. (C and D) Pancreatic histology (H\&E; original magnification, $\times 100$ ) of B16:A-dKO (C) and B16:Y-dKO (D) mice. 


\section{Table 1}

Transplantation of islets and immunization with insulin B:9-23 peptide in B16:A-dK0 mice

\begin{tabular}{lcccc} 
Immunogen & $\begin{array}{c}\text { Pancreatic } \\
\beta \text { cell }\end{array}$ & IAA & Insulitis & Diabetes \\
$\begin{array}{l}\text { Islet transplant } \\
\text { B16:Y } \\
\text { (0/11) }\end{array}$ & B16:A & $85 \%(11 / 13)$ & $0.38 \pm 0.15$ & $0 \%$ \\
$\begin{array}{l}\text { B16:A } \\
\text { Peptide }\end{array}$ & B16:A & $13 \%(1 / 8)$ & $0.09 \pm 0.21$ & $0 \%(0 / 7)$ \\
B16:Y & & & & \\
B16:A & B16:A & $97 \%(29 / 30)$ & $0.11 \pm 0.12$ & $0 \%(0 / 6)$ \\
\hline
\end{tabular}

Colorado at Denver Health Sciences Center (UCDHSC), (Denver, Colorado, USA) Animal Care and Use Committee.

Bone marrow transplantation. Bone marrow was harvested from femurs and tibias of 4-week-old NOD mice, and red blood cells were removed using RBC lysis buffer (Sigma-Aldrich). To deplete mature T and B cells, harvested bone marrow was incubated with $\mathrm{CD}^{+}, \mathrm{CD}^{+}$, and B220 ${ }^{+}$MicroBeads (Miltenyi Biotec), and the labeled cells were magnetically depleted by AutoMACS (Miltenyi Biotec). After cell sorting, we confirmed that the contamination of $\mathrm{CD}^{+}, \mathrm{CD}^{+}$, or $\mathrm{B} 220^{+}$cells was below $5 \%$ by flow cytometric analysis using anti-CD4 (clone H129.19; BD Biosciences - Pharmingen), anti-CD8 (clone 53-6.7; BD Biosciences - Pharmingen), and anti-B220 (clone RA3-6B2; BD Biosciences - Pharmingen) antibodies.

Four-week-old B16:A-dKO and wild-type B16:Y NOD mice received 450 rads of radiation from the cesium irradiator twice at a 4-hour intervals prior to bone marrow injection. T cell- and B cell-depleted bone marrow $\left(1 \times 10^{7}\right.$ cells $)$ was intravenously injected.

Islet transplantation. Pancreatic islets were isolated from adult NOD/SCID or B16:A-dKO mice by collagenase digestion (type V; Sigma-Aldrich) of the pancreas and purification by Histopaque (Sigma-Aldrich). We grafted 4-week-old B16:A-dKO mice with 200 islets beneath the left kidney subcapsular space (45).

Peptide immunization. HPLC-purified B16:Y insulin B:9-23 peptide (SHLVEALYLVCGERG) and the mutated B16:A insulin B:9-23 peptide (SHLVEALALVCGERG) were purchased from Invitrogen. The peptides were dissolved in sterile saline and adjusted to a neutral $\mathrm{pH}$. Four-weekold B16:A-dKO mice were subcutaneously immunized with $100 \mu \mathrm{g}$ of B16: $\mathrm{Y}$ insulin $\mathrm{B}: 9-23$ or B16:A insulin B:9-23 peptide emulsified with CFA.

Adoptive splenocyte transfer experiments. Splenocytes were isolated from B16:A-dKO mice transplanted with either B16:Y or B16:A-dKO islets or immunized with either B16:Y insulin B:9-23 peptide or B16:A insulin B:9-23 peptide, and red blood cells were removed using RBC lysis buffer (Sigma-Aldrich). Splenocytes $\left(3 \times 10^{7}\right)$ were transferred intraperitoneally to 6- to 8-week-old B16:Y NOD/SCID or B16:A-dKO NOD/SCID mice.

For the $\mathrm{CD}^{+} \mathrm{T}$ cell transfer experiment, $\mathrm{CD} 4^{+} \mathrm{T}$ cells or non-CD4 $4^{+} \mathrm{T}$ cells were isolated from splenocytes by depletion of magnetically labeled non-CD4 $4^{+}$ $\mathrm{T}$ cells using CD4 $\mathrm{T}$ cell isolation kit or $\mathrm{CD}^{+} \mathrm{T}$ cells using CD4 MicroBeads, respectively (Miltenyi Biotec). We used AutoMACS (Miltenyi Biotec) for cell sorting. An aliquot of isolated $\mathrm{CD}^{+} \mathrm{T}$ cells or
non-CD4 ${ }^{+} \mathrm{T}$ cells was stained with anti-CD4 antibody (clone H129.19, BD Biosciences - Pharmingen), and the purity was assessed as greater than $95 \%$ by flow cytometric analysis. $\mathrm{CD}^{+}{ }^{+} \mathrm{T}$ cells from peptide-immunized B16:A-dKO mice and non-CD4 ${ }^{+} \mathrm{T}$ cells from nonimmunized B16:A-dKO mice $\left(1.5 \times 10^{7}\right.$ and $4 \times 10^{7}$ cells, respectively) were intraperitoneally transferred to 6 - to 8 -week-old NOD/SCID mice. Similarly, $\mathrm{CD}^{+} \mathrm{T}$ cells from nonimmunized mice and non-CD4 $4^{+} \mathrm{T}$ cells from immunized mice were intraperitoneally transferred to NOD/SCID mice.

Measurement of micro-IAA. B16:A-dKO mice were bled before and every 2 weeks after transplantation or immunization to measure anti-IAAs. Recipient B16:Y NOD/SCID or B16:A-dKO NOD/SCID mice were bled before and weekly after splenocyte transfer. To measure spontaneous development of IAAs, B16:A-dKO and B16:Y-dKO mice were bled every 2-3 weeks between 4 and 30 weeks of age. IAA levels were measured with the 96 -well filtration plate micro-IAA assay previously described (10) and expressed as an index. A value of 0.01 or greater was considered positive.

To investigate the absorption by insulin B:9-23 peptide, $20,000 \mathrm{cpm}$ of human ${ }^{125} \mathrm{I}$-insulin (GE Healthcare) was incubated with $5 \mu$ l of serum overnight along with $100 \mu \mathrm{g} / \mathrm{ml}$ of B16:Y insulin B:9-23 or B16:A insulin B:9-23 peptide, precipitated with protein $A / G$ sepharose (GE Healthcare), and counted in a TopCount beta counter (Packard).

Histology. The pancreata and islet grafts transplanted under kidney capsules were fixed in $10 \%$ formalin and embedded in paraffin. Paraffin-embedded tissue sections were stained with $\mathrm{H} \& \mathrm{E}$, and sections from islet grafts were also stained with polyclonal guinea pig anti-insulin antibodies (Millipore) followed by incubation with a peroxidase-labeled anti-guinea pig IgG antibody (Kierkegaard \& Perry Laboratories Inc.). To evaluate insulitis, more than 10 pancreatic islets from an individual mouse were randomly selected, and each islet was scored as 0 (no insulitis), 0.25 (peri-islet insulitis), and 1 (intra-islet insulitis) by the same reader blinded to the group of mice. The insulitis score was calculated as follows: $([0.25 \times$ no. islets with peri-islet insulitis $]+$ no. islets with intraislet insulitis)/total no. estimated islets.

Diabetes. The blood glucose levels of B16:A-dKO mice were measured every 2 weeks, and recipient NOD/SCID mice that received splenocytes were measured twice per week with the FreeStyle blood glucose monitoring system (TheraSense). Mice were considered diabetic after 2 consecutive blood glucose values greater than $250 \mathrm{mg} / \mathrm{dl}$.

Statistics. The incidence of IAAs and insulitis scores were analyzed with the $\chi^{2}$ test. Survival curves were analyzed with the log-rank test. Statistical tests used PRISM software (version 3.02; GraphPad Software). A $P$ value less than 0.05 was considered significant.
Table 2

Transfer of splenocytes from immunized B16:A-dKO mice to NOD/SCID mice

\begin{tabular}{lccccc}
\hline Immunogen & $\begin{array}{c}\text { Immunized } \\
\text { splenocyte donor }\end{array}$ & $\begin{array}{c}\text { Splenocyte } \\
\text { recipient }\end{array}$ & IAA & Insulitis & Diabetes \\
Islet & B16:A & B16:Y & ND & $0.69 \pm 0.28$ & $83 \%(5 / 6)$ \\
B16:Y & B16:A & B16:Y & ND & $0.07 \pm 0.06$ & $0 \%(0 / 8)^{A}$ \\
B16:A & B16:A & B16:A & ND & $0.26 \pm 0.24$ & $40 \%(2 / 5)$ \\
B16:Y & B16:A & B16:A & ND & 0 & $0 \%(0 / 6)$ \\
B16:A & & & & & \\
Peptide & B16:A & B16:Y & $86 \%(6 / 7)$ & $0.55 \pm 0.28$ & $86 \%(6 / 7)$ \\
B16:Y & B16:A & B16:Y & $0 \%(0 / 8)$ & $0.11 \pm 0.09$ & $0 \%(0 / 8)^{A}$ \\
B16:A & B16:A & B16:A & $0 \%(0 / 6)$ & 0 & $0 \%(0 / 5)$ \\
B16:Y & B16:A & B16:A & $0 \%(0 / 6)$ & 0 & $0 \%(0 / 6)$ \\
B16:A & & & & 0 & \\
\hline
\end{tabular}

ND, not determined. AWithin the group, $25 \%$ (2 of 8 ) were followed up for 35 weeks. ${ }^{\text {BW } W i t h i n ~ t h e ~}$ group, $37 \%$ (3 of 8 ) were followed up for 35 weeks. 


\section{Acknowledgments}

This work was supported by grants from the NIH to G.S. Eisenbarth (DK32083, DK55969, and DK62718) and to E. Liu (DK064605); by a Diabetes Endocrine Research Center grant from the National Institute of Diabetes and Digestive and Kidney Diseases, NIH to G.S. Eisenbarth (P30 DK57516); by a mentor-based fellowship from the American Diabetes Association to M. Kobayshi; by a grant from the Juvenile Diabetes Foundation to G.S. Eisenbarth (JDRF12006-16) and an advanced postdoctoral fellowship to M. Nakayama (JDRF10-2006-51); and by program funds to G.S. Eisenbarth from the Children's Diabetes Foundation.

1. Todd, J.A., Bell, J.I., and McDevitt, H.O. 1987 HLA-DQB gene contributes to susceptibility and resistance to insulin-dependent diabetes mellitus. Nature. 329:599-604.

2. Morel, P.A., et al. 1988. Aspartic acid at position 57 of the HLA-DQ beta chain protects against type I diabetes: a family study. Proc. Natl. Acad. Sci. U. S. A. 85:8111-8115

3. Corper, A.L., et al. 2000. A structural framework for deciphering the link between I-Ag7 and autoimmune diabetes. Science. 288:505-511.

4. Narendran, P., Mannering, S.I., and Harrison, L.C. 2003. Proinsulin - a pathogenic autoantigen in type 1 diabetes. Autoimmun. Rev. 2:204-210.

5. Lieberman, S.M., et al. 2003. Identification of the beta cell antigen targeted by a prevalent population of pathogenic CD8+ T cells in autoimmune diabetes. Proc. Natl. Acad. Sci. U. S. A. 100:8384-8388.

6. Yang, J., et al. 2006. Islet-specific glucose-6phosphatase catalytic subunit-related protein-reactive CD4+ T cells in human subjects. J. Immunol. 176:2781-2789.

7. Jasinski, J.M., and Eisenbarth, G.S. 2005. Insulin as a primary autoantigen for type $1 \mathrm{~A}$ diabetes. Clin. Dev. Immunol. 12:181-186.

8. Pugliese, A. 2005. The insulin gene in type 1 diabetes. IUBMB Life. 57:463-468.

9. Palmer, J.P., et al. 1983. Insulin antibodies in insulin-dependent diabetics before insulin treatment. Science. 222:1337-1339.

10. Yu, L., et al. 2000. Early expression of antiinsulin autoantibodies of humans and the NOD mouse: evidence for early determination of subsequent diabetes. Proc. Natl. Acad. Sci. U. S. A. 97:1701-1706.

11. Alleva, D.G., et al. 2001. A disease-associated cellular immune response in type 1 diabetics to an immunodominant epitope of insulin. J. Clin. Invest. 107:173-180.

12. Pinkse, G.G., et al. 2005. Autoreactive CD8 T cells associated with beta cell destruction in type 1 diabetes. Proc. Natl. Acad. Sci. U. S. A. 102:18425-18430.

13. Wegmann, D.R., Norbury-Glaser, M., and Daniel, D. 1994. Insulin-specific T cells are a predominant component of islet infiltrates in pre-diabetic NOD mice. Eur. J. Immunol. 24:1853-1857.

14. Barker, J.M., et al. 2004. Prediction of autoantibody positivity and progression to type 1 diabetes: Diabetes Autoimmunity Study in the Young (DAISY). J. Clin. Endocrinol. Metab. 89:3896-3902.

15. Bonifacio, E., et al. 2001. International workshop on lessons from animal models for human type 1 diabetes: identification of insulin but not glutamic acid decarboxylase or IA-2 as specific autoantigens of humoral autoimmunity in nonobese diabetic mice. Diabetes. 50:2451-2458.

16. Kent, S.C., et al. 2005. Expanded T cells from pancreatic lymph nodes of type 1 diabetic subjects recognize an insulin epitope. Nature. 435:224-228.

Received for publication December 29, 2006, and accepted in revised form March 20, 2007.

Address correspondence to: George S. Eisenbarth, Barbara Davis Center for Childhood Diabetes, University of Colorado Health Sciences Center, 1775 North Ursula Street, Mail Stop B140, PO Box 6511, Aurora, Colorado 80045-6511, USA. Phone: (303) 724-6847; Fax: (303) 724-6839; E-mail: george.eisenbarth@uchsc.edu.

J.N. Beilke's present address is: Department of Microbiology and Immunology, UCSF, San Francisco, California, USA.

17. Halbout, P., Briand, J.P., Becourt, C., Muller, S., and Boitard, C. 2002. T cell response to preproinsulin I and II in the nonobese diabetic mouse. J. Immunol. 169:2436-2443.

18. Chen, W., et al. 2001. Evidence that a peptide spanning the B-C junction of proinsulin is an early autoantigen epitope in the pathogenesis of type 1 diabetes. J. Immunol. 167:4926-4935.

19. Daniel, D., Gill, R.G., Schloot, N., and Wegmann, D. 1995. Epitope specificity, cytokine production profile and diabetogenic activity of insulin-specific $T$ cell clones isolated from NOD mice. Eur. J. Immunol. 25:1056-1062.

20. Wong, F.S., et al. 1999. Identification of an MHC class I-restricted autoantigen in type 1 diabetes by screening an organ-specific cDNA library. Nat. Med. 5:1026-1031.

21. Yu, L., et al. 1996. Anti-islet autoantibodies develop sequentially rather than simultaneously. J. Clin. Endocrinol. Metab. 81:4264-4267.

22. Ziegler, A.G., Hummel, M., Schenker, M., and Bonifacio, E. 1999. Autoantibody appearance and risk for development of childhood diabetes in offspring of parents with type 1 diabetes: the 2-year analysis of the German BABYDIAB study. Diabetes. 48:460-468.

23. Trudeau, J.D., et al. 2003. Prediction of spontaneous autoimmune diabetes in NOD mice by quantification of autoreactive $T$ cells in peripheral blood. J. Clin. Invest. 111:217-223. doi:10.1172/ JCI200316409.

24. Jaeckel, E., Lipes, M.A., and von Boehmer, H. 2004. Recessive tolerance to preproinsulin 2 reduces but does not abolish type 1 diabetes. Nat. Immunol. 5:1028-1035

25. French, M.B., et al. 1997. Transgenic expression of mouse proinsulin II prevents diabetes in nonobese diabetic mice. Diabetes. 46:34-39.

26. Daniel, D., and Wegmann, D.R. 1996. Protection of nonobese diabetic mice from diabetes by intranasal or subcutaneous administration of insulin peptide B-(9-23). Proc. Natl. Acad. Sci. U. S. A. 93:956-960.

27. Jasinski, J.M., et al. 2006. Transgenic insulin (B:923) T-cell receptor mice develop autoimmune diabetes dependent upon RAG genotype, H-2g7 homozygosity, and insulin 2 gene knockout. Diabetes. 55:1978-1984.

28. Du, W., et al. 2006. TGF- $\beta$ signaling is required for the function of insulin-reactive $\mathrm{T}$ regulatory cells. J. Clin. Invest. 116:1360-1370. doi:10.1172/JCI27030.

29. Nakayama, M., et al. 2005. Prime role for an insulin epitope in the development of type 1 diabetes in NOD mice. Nature. 435:220-223.

30. Faideau, B., et al. 2006. Tolerance to proinsulin-2 is due to radioresistant thymic cells. J. Immunol. 177:53-60.

31. Garcia, C.A., et al. 2005. Dendritic cells in human thymus and periphery display a proinsulin epitope in a transcription-dependent, capture-independent fashion. J. Immunol. 175:2111-2122.

32. Steptoe, R.J., Ritchie, J.M., Jones, L.K., and Harrison, L.C. 2005. Autoimmune diabetes is suppressed by transfer of proinsulin-encoding $\mathrm{Gr}-1(+)$ myeloid progenitor cells that differentiate in vivo into resting dendritic cells. Diabetes. 54:434-442.

33. Pugliese, A. 2004. Central and peripheral autoantigen presentation in immune tolerance. Immunology. 111:138-146.

34. Ouyang, Q., et al. 2006. Recognition of HLA class I-restricted beta-cell epitopes in type 1 diabetes. Diabetes. 55:3068-3074.

35. Krishnamurthy, B., et al. 2006. Responses against islet antigens in NOD mice are prevented by tolerance to proinsulin but not IGRP. J. Clin. Invest. 116:3258-3265. doi:10.1172/JCI29602.

36. Gallucci, S., and Matzinger, P. 2001. Danger signals: SOS to the immune system. Curr. Opin. Immunol. 13:114-119.

37. Wong, F.S., Moustakas, A.K., Wen, L., Papadopoulos, G.K., and Janeway, C.A., Jr. 2002. Analysis of structure and function relationships of an autoantigenic peptide of insulin bound to $\mathrm{H}-2 \mathrm{~K}(\mathrm{~d})$ that stimulates CD8 $\mathrm{T}$ cells in insulin-dependent diabetes mellitus. Proc. Natl. Acad. Sci. U. S. A. 99:5551-5556.

38. Noorchashm, H., Greeley, S.A., and Naji, A. 2003. The role of $\mathrm{T} / \mathrm{B}$ lymphocyte collaboration in the regulation of autoimmune and alloimmune responses. Immunol. Res. 27:443-450.

39. Abiru, N., et al. 2001. Peptide and major histocompatibility complex-specific breaking of humoral tolerance to native insulin with the B9-23 peptide in diabetes-prone and normal mice. Diabetes. 50:1274-1281.

40. Simone, E., et al. 1997. T cell receptor restriction of diabetogenic autoimmune NOD T cells. Proc. Natl. Acad. Sci. U. S. A. 94:2518-2521.

41. Harashima, S., Clark, A., Christie, M.R., and Notkins, A.L. 2005. The dense core transmembrane vesicle protein IA-2 is a regulator of vesicle number and insulin secretion. Proc. Natl. Acad. Sci. U. S. A. 102:8704-8709.

42. Nakayama, M., et al. 2004. The establishment of native insulin-negative NOD mice and methodology to distinguish specific insulin knockout genotypes and a B:16 alanine preproinsulin transgene. Ann. N. Y. Acad. Sci. 1037:193-198.

43. Duvillié, B., et al. 1997. Phenotypic alterations in insulin-deficient mutant mice. Proc. Natl. Acad. Sci. U. S. A. 94:5137-5140.

44. Moriyama, H., et al. 2003. Evidence for a primary islet autoantigen (preproinsulin 1) for insulitis and diabetes in the NOD mouse. Proc. Natl. Acad. Sci. U. S. A. 100:10376-10381.

45. Nicolls, M.R., Coulombe, M., Beilke, J., Gelhaus, H.C., and Gill, R.G. 2002. CD4-dependent generation of dominant transplantation tolerance induced by simultaneous perturbation of CD154 and LFA-1 pathways. J. Immunol. 169:4831-4839. 